\title{
ALGORITHM FOR DECOUPLING AND COMPLETE POLE ASSIGNMENT OF LINEAR MULTIVARIABLE SYSTEMS*
}

\author{
J.C. Zúñiga†, J. Ruiz-León $\ddagger$, and D. Henrion $\dagger \S^{\dagger}$ \\ $\dagger$ Laboratoire d'Analyse et d'Architecture des Systèmes, \\ Centre National de la Recherche Scientifique \\ 7 Avenue du Colonel Roche \\ 31077 Toulouse, cedex 4, France \\ E-mail: jczuniga@laas.fr, henrion@laas.fr \\ §Institute of Information Theory and Automation \\ Academy of Sciences of the Czech Republic, Prague. \\ $\ddagger$ CINVESTAV-IPN, Unidad Guadalajara. \\ P.O. Box 31-438, Plaza la Luna. \\ 44550 Guadalajara, Jalisco. Mexico. \\ E-mail: jruiz@gdl.cinvestav.mx
}

Keywords : Numerical algorithms; linear systems; decoupling; infinite structure; pole assignment.

\begin{abstract}
The problem of decoupling and complete pole assignment of linear square, and controllable systems by static state feedback is addressed in this paper. Based on a characterization of the whole set of attainable finite pole-zero structures of a decouplable system, we present a reliable numerical algorithm which tests the conditions for decoupling and computes the state feedback which decouples the system with a particular pole-zero finite structure, avoiding unnecessary cancellations of invariant zeros. With the use of this algorithm, fixed decoupling poles are determined, non-fixed poles can be arbitrarily located, and no cancellation of system invariant zeros is produced, if this is not necessary for decoupling.
\end{abstract}

\section{Introduction}

We are interested in this work in the row-by-row decoupling of linear multivariable systems with the same number of inputs and outputs (square systems) by static state feedback. The solution to this problem was obtained in [6], based on the nonsingularity of a matrix constructed from the system matrices; the solution in terms of the

*J. C. Zúñiga acknowledges support from the National Council of Science and Technology of Mexico (CONACYT) and from the Secretariat of Public Education of Mexico (SEP). D. Henrion acknowledges support from the Grant Agency of the Czech Republic under Project No. 102/02/0709.

${ }^{\dagger}$ Corresponding author is D. Henrion. Fax: +33 561336969 . E-mail: henrion@laas.fr infinite structure of the system is presented in [5]. The decoupling problem with stability of square systems has been solved in [13] using a geometric approach, and in [14] using a polynomial equation approach. Even though there exist many results concerning this problem, most of the contributions in the literature about decoupling focus mainly on the necessary and sufficient conditions to solve the problem, but they usually do not consider neither the issue of what the structure of the decoupled closed-loop system may be (aside from the diagonality of the closedloop transfer function matrix) nor the characteristics of the decoupling state feedback. Actually, in order to simplify the problem, a common consideration is that the entries of the closed-loop transfer function matrix are supposed to be of the form $1 / s^{j}$, where $j$ is a positive integer, which is also referred to as integrator decoupling. Of course, no pole locations to obtain adequate system dynamics are considered within this approach, not to speak of the problems (for instance, internal stability) which may be caused by possible pole-zero cancellations. Achieving first decoupling, for example in integrator decoupling form, and after that trying to assign the poles of the system can be a difficult problem, since the state feedback designed to solve the pole-assignment will usually destroy the diagonality of the closed-loop transfer function matrix. In light of this, a more reasonable approach seems to be to achieve both objectives using the same state feedback. Considering pole-zero cancellations, it is well known that in order to decouple a linear system, it may be necessary to cancel some invariant zeros of the system with closed-loop poles, but that not necessarily all invariant system zeros have to be cancelled. Then, a complete pole assignment for the decoupling problem should provide the whole set of finite pole-zero structures which can be obtained for the closed-loop sys- 
tem, avoiding unnecessary cancellations of invariant zeros.

As far as the structure of a decoupled closed-loop system is concerned, a first attempt to study this structure was presented in [6], where the authors characterized the class of all feedback matrices which decouple a system, and the number of closed-loop poles which can be assigned. Their conditions, however, are cumbersome and difficult to apply, there is no connection whatsoever of these conditions to the structure of the system, and they show how to assign only a number of poles equal to the sum of the system infinite zero orders, which is in general less than the true number of assignable poles. The problem of decoupling and pole assignment is tackled in [19] using a geometric approach, and the authors present necessary and sufficient conditions to solve this problem based on the concept of controllability subspaces and their properties. Fixed decoupling poles are proved in [12] to be equal to the interconnection transmission zeros, as defined in this reference. The characterization of the closed-loop structure of a decouplable system, and the properties of the decoupling state feedback are presented in [16]. In this reference, the family of all attainable transfer function matrices for the decoupled closed-loop system is determined, which also establishes all possible combinations of finite closed-loop pole and zero structures.

Concerning numerical algorithms related to decoupling, a reliable numerical algorithm for the computation of the interactor of a linear multivariable system is presented in [15]. Avoiding of use of elementary operations, the algorithm uses only reliable operations from numerical linear algebra, and it is further used to compute the state feedback which decouples a linear multivariable system, producing the inverse of the system interactor as closed-loop transfer function matrix. In [4], a numerical method for proportional and derivative state-feedback decoupling controller design is presented, based on orthogonal transformations. Solvability conditions of all solutions for the triangular decoupling problem are presented in [3], based on condensed forms obtained from orthogonal transformations. In [2] it is presented an algorithm for the decoupling problem with stability, based also on orthogonal transformations and condensed forms. The reported algorithm computes a state feedback which solves the decoupling problem with stability.

The aim of this paper is to develop a reliable numerical method to address the problem of decoupling and complete pole assignment of linear multivariable systems. We present in this work a reliable numerical algorithm which tests the conditions for the decouplability of a linear, multivariable, square and controllable system, and computes the corresponding state feedback which decouples the system with a particular pole-zero finite structure. From the previously mentioned references on numerical algorithms related to decoupling, the closest to our present work is [2]. Making a comparison, it can be said that the problem we are considering in this work is more general than that of [2], in the sense that we provide a numerically reliable algorithm to compute a state feedback which not only decouples the system, but also determines the fixed decoupling poles and allows a complete pole assignment avoiding unnecessary cancellation of invariant zeros. Decoupling with stability is indeed a particular case of this more general setting. The tools on which the numerically algorithms are based are also different from those used in [2].

In this work, we first extend the results presented in [16] to drop the observability assumption, obtaining a complete characterization of the closed-loop structure of a controllable and decouplable system, not necessarily observable. From this result, fixed decoupling poles can be determined, as well as the complete finite pole-zero structure which can be obtained for the decoupled system.

We then develop a numerical algorithm based on these results to solve the decoupling and complete pole assignment problem. The relevant information (global and row infinite zeros, and global and row finite zeros of the system) is obtained in a numerically reliable way from the Kronecker invariants of suitable matrix pencils. Given a particular attainable finite pole-zero structure for the closed-loop system, the algorithm computes the state feedback with decouples the system with this pole-zero structure, from the constant kernel of a polynomial matrix. Besides testing the conditions for decoupling and computing the state feedback which solves the problem, the algorithm also determines the fixed decoupling poles of the system, which are fundamental, for instance, in the issue of internal stability. Cancellation of invariant zeros of the system is completely avoided if it is not necessary for decoupling. The algorithm described in the paper will be implemented in the new release of the Polynomial Toolbox for MATLAB ${ }^{1}$.

Some preliminaries are introduced in Section 2, the characterization of the closed-loop structure of a decouplable system is presented in Section 3, and the algorithm for decoupling and complete pole assignment is presented in Section 4. An illustrative example is presented in Section 5 , and we end up with some conclusions.

\section{Preliminaries}

We consider in this work linear multivariable systems with the same number of inputs and outputs, described by

$$
(A, B, C)\left\{\begin{array}{l}
\dot{x}(t)=A x(t)+B u(t) \\
y(t)=C x(t)
\end{array}\right.
$$

${ }^{1}$ MATLAB is a trademark of The MathWorks Inc.; for the Polynomial Toolbox, see www.polyx.cz. 
where $x \in \mathbb{R}^{\mathrm{n}}, u \in \mathbb{R}^{\mathrm{m}}$ and $y \in \mathbb{R}^{\mathrm{m}}$ are, respectively, the state, input and output vectors of the system.

The system $(A, B, C)$ is said to be row by row decouplable by static state feedback if there exists a state feedback

$$
(F, G): \quad u(t)=F x(t)+G v(t),
$$

where $F \in \mathbb{R}^{\mathrm{m} \times \mathrm{n}}$ and $G \in \mathbb{R}^{\mathrm{m} \times \mathrm{m}}$ are constant matrices, with $G$ nonsingular, and $v(t)$ is a new input vector, such that the input $v_{i}(t)$ controls the output $y_{i}(t)$, $i=1, \ldots, m$, without affecting the other outputs.

From the input-output point of view, the previous formulation is equivalent to the existence of a state feedback $(F, G)$ such that the transfer function matrix $T_{F, G}(s)$ of the closed-loop system $(A+B F, B G, C)$ is a nonsingular diagonal matrix, i.e.,

$$
\begin{aligned}
T_{F, G}(s) & =C(s I-A-B F)^{-1} B G \\
& =\operatorname{diag}\left\{w_{1}(s), \ldots, w_{m}(s)\right\}=: W(s)
\end{aligned}
$$

where $w_{i}(s) \neq 0, i=1, \ldots, m$, are strictly proper rational functions.

If the stability issue is considered in the problem formulation, then the system $(A, B, C)$ is said to be decouplable with stability if it is decouplable and the closed-loop system $(A+B F, B G, C)$ is internally stable, i.e., if the eigenvalues of the matrix $(A+B F)$ are located in the open left half complex plane.

The conditions for decoupling a linear multivariable system $(A, B, C)$ are intimately connected to the structure of the system matrix

$$
P(s)=\left[\begin{array}{cc}
s I-A & B \\
C & 0
\end{array}\right]
$$

related to the structure of the matrices

$$
P_{i}(s)=\left[\begin{array}{cc}
s I-A & B \\
c_{i} & 0
\end{array}\right], i=1, \ldots, m,
$$

where $c_{i}$ is the $\mathrm{i}$-th row of matrix $C, i=1, \ldots, m$.

Indeed, the system $(A, B, C)$ is decouplable if and only if the infinite structure of $P(s)$ coincides with the infinite structure of the matrices $P_{i}(s)$, i.e., if and only if

$$
\sum_{i=1}^{m} n_{i}^{\prime}=\sum_{i=1}^{m} n_{i}
$$

where $\left\{n_{1}^{\prime}, \ldots, n_{m}^{\prime}\right\}$ are the infinite zero orders of $P(s)$ (infinite zero orders of the system), and $\left\{n_{1}, \ldots, n_{m}\right\}$ are the infinite zero orders of $P_{1}(s), \ldots, P_{m}(s)$ (row infinite zero orders of the system) $[6,5]$.
If the system is decouplable, then it is decouplable with stability if and only if the number of unstable zeros of $P(s)$ (unstable zeros of the system), multiplicities included, is equal to the number of unstable zeros of $P_{1}(s), \ldots, P_{m}(s)$ (row unstable zeros of the system), taken all together $[13,14]$.

\section{Characterization of the closed- loop structure}

It is well known that in the process of decoupling a linear system, some of the finite zeros of the system may be cancelled by assigning closed-loop poles to the position of finite zeros. It is important, however, to make the distinction between finite zeros that must be cancelled in order to achieve decoupling, and finite zeros which are not necessary to cancel. In practical designs, cancellation of finite zeros is usually avoided because of potential internal instability caused by hidden system dynamics and undesirable pole locations, which is dramatically important in the case of unstable finite zeros. Thus, if the main objective is to decouple the system, it is important at least to know the number of finite poles which can be freely assigned, and the number of poles which have to be cancelled with finite zeros in order to achieve decoupling, i.e., the so-called fixed decoupling poles. ${ }^{2}$ This information would provide a complete characterization of the closed-loop structure and complete pole assignment of a decouplable system.

Concerning non-observable systems, instead of finite zeros it is necessary to consider the invariant zeros of the system, which may also appear or not as finite zeros of the closed-loop decoupled system. The characterization of the closed-loop structure of a square decouplable and observable system presented in [16] can be extended to consider the case of non-observable systems as follows.

First, let us introduce the following preliminary result.

Lemma 1. Let $(A, B, C)$ be a square controllable system, and let $c_{i}$ be the $\mathrm{i}$-th row of matrix $C, i=1, \ldots, m$. Then, the matrix

$$
P_{i}(s)=\left[\begin{array}{cc}
s I-A & B \\
c_{i} & 0
\end{array}\right]
$$

can have at most one non-unit invariant polynomial.

Proof. The invariant polynomials of $P_{i}(s)$ can be obtained

\footnotetext{
${ }^{2}$ Strictly speaking, cancelled frequency values are not system poles, since they do not appear in the system transfer function matrix. Then, it should be more appropriate to speak of fixed decoupling modes, where poles are a subset of the system modes, and both sets are equal if the system is controllable and observable. For simplicity, we made no distinction in this paper between modes and poles.
} 
as

$$
\lambda_{j}(s)=\frac{\Delta_{j}(s)}{\Delta_{j-1}(s)}, \quad j=1, \ldots, n+1,
$$

where

$$
\begin{aligned}
& \Delta_{0}(s):=0, \\
& \Delta_{j}(s)=\operatorname{gcd} \text { of all } j \times j \text { minors of } P_{i}(s),
\end{aligned}
$$

are the determinantal divisors of $P_{i}(s)$ (see for instance [11]). Since the system is controllable, at least the first $n$ determinantal divisors of $P_{i}(s)$ are all units. Then, the only possible non-unit invariant polynomial of $P_{i}(s)$ is the last one, which is equal to $\Delta_{n+1}(s)$.

Let us denote as $z_{i}(s)$ the last invariant polynomial of matrix $P_{i}(s), i=1, \ldots, m$. The finite row zeros of the system is a subset of the roots of $z_{i}(s)$, and both sets coincide if $(A, B, C)$ is observable. It can be seen than any finite zero of $P_{i}(s)$ is also a zero of the matrix $P(s)$ given by (2), but that a zero of $P(s)$ is not necessarily a zero of $P_{i}(s)$. Then the product of the polynomials $\prod_{i=1}^{m} z_{i}(s)$ divides exactly $\prod_{i=1}^{n+m} \epsilon_{i}(s)$, where $\epsilon_{i}(s)$ are the invariant polynomials of $P(s)$.

The family of all attainable transfer function matrices for the decoupled closed-loop system is characterized by the following result.

Theorem 1. Let $(A, B, C)$ be a square, controllable, and decouplable system. Then, there exists a state feedback $(F, G)$ which decouples the system, such that the transfer function of the decoupled closed-loop system is of the form

$$
\begin{aligned}
W(s) & =C(s I-A-B F)^{-1} B G \\
& =\left[\begin{array}{ccc}
k_{1} \frac{z_{1}(s)}{a_{1}(s)} & & \\
& \ddots & \\
& & k_{p} \frac{z_{m}(s)}{a_{p}(s)}
\end{array}\right]
\end{aligned}
$$

where $k_{1}, \ldots, k_{m}$ are real numbers, $z_{i}(s)$ is the last invariant polynomial of matrix $P_{i}(s), i=1, \ldots, m$, as introduced before, $a_{1}(s), \ldots, a_{m}(s)$ are monic polynomials with arbitrary roots, satisfying

$$
\operatorname{deg} a_{i}(s)-\operatorname{deg} z_{i}(s)=n_{i}, \quad i=1, \ldots, m,
$$

and $n_{1}, \ldots, n_{m}$ are the row infinite zero orders of the system.

Proof. The proof is essentially the same as in Theorem 1 of [16]. Just observe that if a preliminary state feedback is applied to render the system observable, then the roots of $z_{i}(s)$ become the row finite zeros of the system.

Theorem 1 completely characterizes the set of all matrices which can be obtained as closed-loop transfer function matrices of a decouplable system. This characterization provides also all the set of possible finite pole-zero structure for the closed-loop system. Indeed, it can be seen that the finite zeros and poles of the closed-loop system are respectively given by the roots of numerator polynomials $z_{i}(s)$ and denominator polynomials $a_{i}(s)$ of $W(s)$.

Theorem 2. Fixed decoupling poles of the system correspond to the roots of the polynomial

$$
\delta(s):=\frac{\prod_{i=1}^{n+m} \epsilon_{i}(s)}{\prod_{i=1}^{m} z_{i}(s)}
$$

where $\epsilon_{1}(s), \ldots, \epsilon_{n+m}(s)$ are the invariant polynomials of $P(s)$, and $z_{i}(s)$ is the last invariant polynomial of $P_{i}(s)$, $i=1, \ldots, m$.

Proof. The set of invariant zeros of $(A, B, C)$ are the roots of the polynomials $\epsilon_{i}(s)$, and it is evident from (5) that the only frequency values that can be finite zeros of the decoupled closed-loop system are the roots of the polynomials $z_{i}(s)$. If $\delta(s)$ is not a unit, then some of the poles of the system (the fixed decoupling poles) must be located at the positions of the roots of $\delta(s)$ producing cancellation with finite zeros of the system.

Remark 1. From the previous result, it can be seen that the fixed decoupling poles correspond to invariant zeros which are not row invariant zeros of the system. Observe also that there are no fixed decoupling poles (all system poles can be assigned) if the system has no invariant zeros, or if all invariant zeros (multiplicities included) are also row invariant zeros of the system.

Remark 2. It follows from Theorem 2 that the number of poles which can be arbitrarily assigned while decoupling the system is equal to

$$
n-\operatorname{deg} \delta(s) \text {, }
$$

where $n$ is the order of the system and $\delta(s)$ is given by (7).

\section{Algorithm for decoupling and pole assignment}

Based on the results from Section 3, we develop in this section a numerical algorithm to solve the decoupling and complete pole assignment problem. The relevant information is the global and row infinite zeros, and global and row invariant finite zeros of the system. It is well known that computing the finite or infinite structure of a pencil from its Smith form is not numerically reliable. Instead of that, we will obtain this information in a numerically reliable way from the Kronecker invariants of suitable matrix pencils. The algorithm for that purpose is based on the results from [17, 18] and uses only numerically reliable operations such as Householder 
transformations or the singular value decomposition (SVD), see for instance $[7,8]$.

Let us consider an arbitrary $m \times n$ matrix $A$ and compute its SVD,

$$
P^{T} A Q=\Sigma
$$

where $\Sigma$ is an $m \times n$ matrix with singular values of $A$ along its main diagonal. The rank $r$ of $A$ corresponds to the number of non-zero singular values, and we have that

$$
\begin{aligned}
& P^{T} A=\left[\frac{A_{r}}{0}\right], \\
& A Q=\left[A_{c} \mid 0\right]
\end{aligned}
$$

where $A_{r}$ and $A_{c}$ have $r$ linearly independent rows and columns respectively. Operation $(9 a)$ is called row compression and operation (9b) column compression of A.

Consider an arbitrary $m \times n$ pencil $\mathcal{P}(s)=s E-L$. The algorithm to obtain the eigenstructure of $\mathcal{P}(s)$ is described as follows (see [17]).

- Let $E_{1}=E, n_{1}=n, m_{1}=m$ and $L_{1}=L$.

- Step $k$ : Obtain the SVD $\Sigma=P^{T} E_{k} Q$ and the rank $\rho_{k}$ of $L_{k}$. If $v_{k}=m_{k}-\rho_{k}$ is not zero, make the permuted row compressions.

$$
I_{p} P^{T} E_{k}=\left[\begin{array}{c}
0 \\
E_{k}
\end{array}\right], \quad I_{p} P^{T} L_{k}=\left[\begin{array}{c}
\bar{L}_{k} \\
L_{k}
\end{array}\right],
$$

where $I_{p}=\left[\begin{array}{cc}0 & I_{\rho_{k}} \\ I_{v_{k}} & 0\end{array}\right]$.

Obtain the SVD $S=P^{T} \bar{L}_{k} Q$ and the rank $r_{k}$ of $\bar{L}_{k}$, and make the column compressions

$$
\bar{L}_{k} Q=[* \mid 0], \quad E_{k} Q=\left[* \mid E_{k+1}\right], \quad L_{k} Q=\left[* \mid L_{k+1}\right] .
$$

where irrelevant entries are denoted by stars.

- Update the dimensions

$$
m_{k+1}=m_{k}-v_{k}, \quad n_{k+1}=n_{k}-r_{k}
$$

and go to next step $k+1$ (notice that in each compression matrices $E_{k}$ and $L_{k}$ are modified).

If $v_{k}$ is zero, structural indices at infinity of $\mathcal{P}(s)$ can be recovered from vectors $v=\left[v_{1}, \ldots, v_{k}\right]$ and $r=\left[r_{1}, \ldots, r_{k-1}\right]$. For $i=1,2, \ldots, k-1, \mathcal{P}(s)$ has $r_{i}-v_{i+1}$ zeros at infinity of degree $i-1$. In addition, it can be shown that the $m_{k} \times n_{k}$ pencil $s E_{k}-L_{k}$ contains only the finite structure and the right null space of $\mathcal{P}(s)$.

Now we take the reduced pencil $s E_{k}-L_{k}$ and apply a dual process, namely, we start with the column compressions

$$
E_{k} Q=\left[E_{k} \mid 0\right], \quad L_{k} Q=\left[L_{k} \mid \bar{L}_{k}\right],
$$

and apply the row permuted compressions

$$
\begin{gathered}
I_{p} P^{T} \bar{L}_{k}=[0], \quad I_{p} P^{T} E_{k}=\left[\begin{array}{c}
* \\
E_{k+1}
\end{array}\right], \\
I_{p} P^{T} L_{k}=\left[\begin{array}{c}
* \\
L_{k+1}
\end{array}\right],
\end{gathered}
$$

update the dimensions

$$
n_{k+1}=n_{k}-v_{k}, \quad m_{k+1}=m_{k}-r_{k}
$$

and go to the next step.

When the dual process is finished, the new square reduced pencil $s \widehat{E}-\widehat{L}$ contains only the finite zeros of $\mathcal{P}(s)$.

Then, using the well known QZ factorization

$$
Q \widehat{E} Z=\widetilde{E}, Q \widehat{L} Z=\widetilde{L},
$$

the finite zeros can be obtained as ratios of diagonal elements $\alpha_{i}=\widetilde{l}_{i i} / \widetilde{e}_{i i}$. The QZ factorization is also based on Householder transformations (see [7]).

The above method allows to obtain the eigenstructure of a given pencil in a numerically reliable way. Then, we can easily obtain the global and row, finite and infinite zeros of the system $(A, B, C)$ from the eigenstructure of the pencils given by (2) and (3). In this way, the conditions (4) for decoupling can be tested, and the whole set of attainable finite pole-zero structures for the decoupled closed-loop system can be characterized.

To complete the algorithm, we will show how to compute the state feedback which produces a decoupled closed-loop system with a particular finite pole-zero structure, also in a numerically reliable way.

It is shown in [16] that for a particular choice of closed-loop transfer function matrix from the set (5), say $W_{1}(s)$, the corresponding state feedback $(F, G)$ producing $W_{1}(s)$ is unique if and only if the system is controllable. Instead of the proposed method of [16] to obtain $(F, G)$ from the constant solution of a polynomial matrix equation, we will use the following method, which is more suitable to be implemented using reliable numerical routines, and has the advantage in comparison to the one of [16] that it is not necessary to obtain a matrix fraction description $N(s), D(s)$ of the system with $D(s)$ column reduced.

Let $W_{1}(s)$ be a particular transfer function matrix from the set (5), and define

$$
Q(s)=T^{-1}(s) W(s)=Q_{0}+\bar{Q}(s),
$$

where $T(s)$ is the transfer function of the system, $Q_{0}$ is a constant matrix, and $\bar{Q}(s)$ is a strictly proper rational 
matrix.

Then, we seek matrices $F$ and $G$ such that

$$
Q(s)=\left[I-F(s I-A)^{-1} B\right]^{-1} G .
$$

From the last equation, it can be seen that matrix $G$ is given by

$$
G=\lim _{s \rightarrow \infty} Q(s)=Q_{0} .
$$

To compute matrix $F$, let $\left[\begin{array}{ll}L & E\end{array}\right]$ be a basis for the left constant kernel of the matrix

$$
\left[\begin{array}{l}
(s I-A)^{-1} B \\
I-G Q^{-1}(s)
\end{array}\right]
$$

where $L \in \mathbb{R}^{\mathrm{m} \times \mathrm{n}}, E \in \mathbb{R}^{\mathrm{m} \times \mathrm{m}}$, and $E$ is nonsingular (such matrices always exist since the system is decouplable). Observe that the left constant kernel is not modified if matrix (11) is transformed in

$$
\left[\begin{array}{c}
\operatorname{det}(Q) \operatorname{adj}(s I-A) B \\
\operatorname{det}(s I-A)(\operatorname{det}(Q) I-G \operatorname{adj}(Q))
\end{array}\right]
$$

using the reliable methods to compute the adjoint of a polynomial matrix presented in $[9,10]$. Thus, this information can be obtained from the constant kernel of a polynomial matrix. To this end, we use numerical reliable routines based on Householder transformations (see [1]).

Finally, the matrix $F$ satisfying

$$
C(s I-A-B F)^{-1} B G=W_{1}(s)
$$

is given by

$$
F=-L E^{-1}
$$

\section{Example}

The following example illustrates our results. Actually, this example is presented more to illustrate the issues of pole assignment and cancellation or not of invariant zeros, than the numerical properties of the algorithm, which were also tested with different examples, but that are not presented here.

Example 1. Let the controllable system $(A, B, C)$ be given by

$$
\begin{gathered}
A=\left[\begin{array}{rrrrr}
-2 & 3 & 0 & -1 & 1 \\
1 & 0 & 0 & 0 & 0 \\
-2 & -1 & -1 & 3 & 5 \\
0 & 0 & 1 & 0 & 0 \\
0 & 0 & 0 & 1 & 0
\end{array}\right], \\
B=\left[\begin{array}{rr}
0 & 1 \\
0 & 0 \\
-1 & 1 \\
0 & 0 \\
0 & 0
\end{array}\right], C=\left[\begin{array}{rrrrr}
0 & 1 & 0 & -1 & -1 \\
1 & -1 & 0 & 0 & 0
\end{array}\right]
\end{gathered}
$$

whose transfer function is

$$
T(s)=\left[\begin{array}{cc}
\frac{1}{(s-2)(s+2)} & 0 \\
\frac{s-1}{(s-2)(s+2)^{3}} & \frac{s+1}{(s+2)^{2}}
\end{array}\right] .
$$

Applying the reported algorithm, we obtain the following results, which can be easily checked: the system is decouplable, the set of matrices which can be obtained as transfer function matrices for the decoupled closed-loop system is given by

$$
W(s)=\left[\begin{array}{cc}
\frac{k_{1}}{\left(s+\alpha_{1}\right)\left(s+\alpha_{2}\right)} & 0 \\
0 & \frac{k_{2}(s-1)}{\left(s+\alpha_{3}\right)\left(s+\alpha_{4}\right)}
\end{array}\right] .
$$

and there exists a fixed decoupling pole at $s=-1$. Notice that the system is decouplable with stability, since there are no fixed unstable poles, and that 4 out of the 5 system poles can be arbitrarily assigned. Observe also that $s=1$ is an invariant row and global zero of the system, which is not evident from the system transfer function, since the system is not observable; thus, this zero can also appear in $W(s)$ using a state feedback which decouples the system.

Let us choose a pole-zero finite structure corresponding to the following matrix

$$
W_{1}(s)=\left[\begin{array}{cc}
\frac{1}{(s+1)(s+2)} & 0 \\
0 & \frac{s-1}{(s+2)^{2}}
\end{array}\right]
$$

i.e. we want to obtain a decoupled and internally stable closed-loop system, with poles at the positions specified by $W_{1}(s)$.

The unique state feedback producing $W_{1}(s)$ is computed by the algorithm as

$$
F=\left[\begin{array}{rrrrr}
-3 & -6 & 3 & 9 & 6 \\
-2 & -7 & 0 & 1 & -1
\end{array}\right], \quad G=\left[\begin{array}{ll}
1 & 0 \\
0 & 1
\end{array}\right]
$$

\section{Conclusions}

We presented in this paper a reliable numerical algorithm which tests the conditions for decoupling and computes the state feedback which decouples the system with a particular pole-zero finite structure, avoiding unnecessary cancellations of invariant zeros.

It is well known that small variations on the values of the system matrices of a perfectly decouplable system, due for instance to rounding errors, can lead to the wrong conclusion that the system is not decouplable, or not decouplable with a particular structure (see for instance Example 2 in [2]). This is a problem of the system representation, and not of the reliability or stability of the decoupling algorithm itself. Actually, any reliable 
algorithm would give the same results. Concepts of wellposedness, genericity, and results on "robust decoupling" have to be considered to give an answer to this problem.

Numerical testings, which are not included in this paper, demonstrated that the algorithm performs quite well. Both numerical error analysis and computational complexity of the algorithm remain to be studied.

\section{References}

[1] Beelen T.G.J., and Weltkamp G.W., "Numerical computation of a coprime factorization of a transfer function matrix", Systems and Control Letters, $\mathbf{9}$, 281-288 (1987).

[2] Chu D., and Tan R.C.E, "Numerically reliable computing for the row by row decoupling problem with stability", SIAM J. Matrix Anal. Appl., 23, 11431170 (2002).

[3] Chu D., and Tan R.C.E, "Solvability conditions and parameterization of all solutions for the triangular decoupling problem", SIAM J. Matrix Anal. Appl., 23, 1171-1182 (2002).

[4] Chu D., and Malabre M., "Numerically reliable design for proportional and derivative state-feedback decoupling controller", Automatica, 38, 2121-2125 (2002).

[5] Descusse J., and Dion J.M., "On the structure at infinity of linear square decoupled systems" IEEE Trans. Automat. Contr., AC-27, 971-974 (1982).

[6] Falb P.L., and Wolovich W.A., "Decoupling in the design and synthesis of multivariable control systems", IEEE Trans. Automat. Contr., AC-12, 651659 (1967).

[7] Golub G.H., and Van Loan C.F., Matrix Computations, The Johns Hopkins University Press, New York, (1996).

[8] Higham N.J., Accuracy and Stability of Numerical Algorithms, SIAM, Philadelphia, (1996).

[9] Hromčík M., and Šebek M., "New algorithm for polynomial matrix determinant based on FFT", Proc. European Control Conference, Karlsruhe, Germany (1999).

[10] Hromčík M., and Šebek M., "FFT and linear matrix polynomial equations", Proc. IFAC Symposium on Systems Structure and Control, Prague, Czech Republic (2001).

[11] Kailath T., Linear Systems, Prentice Hall, Englewood Cliffs, N.J., (1980).

[12] Koussiouris T.G., "A frequency domain approach for the block decoupling problem II. Pole assignment while block decoupling a minimal system by state feedback and a constant non-singular input transformation and observability of the block decoupled system", Int. J. of Control, 32, 443-464 (1980).
[13] Martinez J.C., and Malabre M., "The row by row decoupling problem with stability: A structural approach", IEEE Trans. Automat. Contr., 39, 24572460 (1994).

[14] Ruiz-León J., Zagalak P., and Eldem V., "On the Morgan problem with stability", Kybernetika, 32, 425-441 (1996).

[15] Ruiz-León J., Zúñiga J.C., and Henrion D., "Computation of the interactor of a linear multivariable system", Proc. IASTED International Conference on Control and Applications, 285-288, Banff, Canada (2001).

[16] Ruiz-León J., Orozco J.L., and Begovich O., "On the structure of the closed-loop system in the decoupling of linear multivariable systems", accepted to the Latin-American Conference on Automatic Control CLCA 2002, Guadalajara, Mexico, to be held on December 2002.

[17] Van Dooren P., "The computation of Kronecker's canonical form of a singular pencil", Linear Algebra an its Applications, 27, 103-141 (1979).

[18] Van Dooren P., and Dewilde P., "The eigenstructure of an arbitrary polynomial matrix: Computational aspects", Linear Algebra and its Applications, 50, 545-580 (1983).

[19] Wonham W.M., and Morse A.S., "Decoupling and pole assignment in linear multivariable systems: A geometric approach", SIAM J. Contr., 8, 1-18 (1970). 